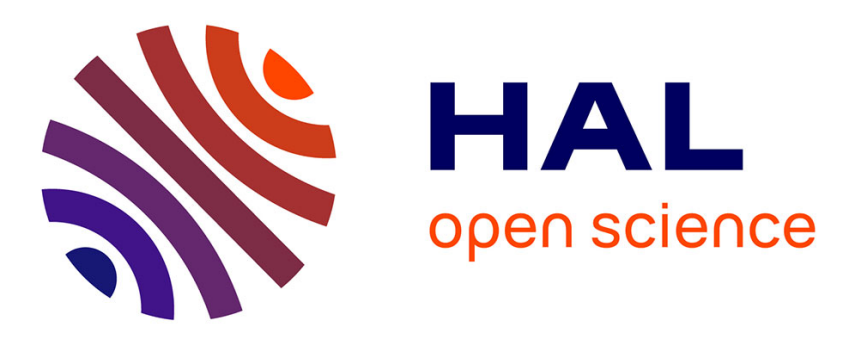

\title{
Reduction of quantum noise in optical parametric amplification
}

Juan Ariel Levenson, Izo Abram, Thomas Rivera, Philippe Grangier

\section{To cite this version:}

Juan Ariel Levenson, Izo Abram, Thomas Rivera, Philippe Grangier. Reduction of quantum noise in optical parametric amplification. Journal of the Optical Society of America B, 1993, 10 (11), pp.2233-2238. hal-00870996

\section{HAL Id: hal-00870996 \\ https://hal-iogs.archives-ouvertes.fr/hal-00870996}

Submitted on 8 Oct 2013

HAL is a multi-disciplinary open access archive for the deposit and dissemination of scientific research documents, whether they are published or not. The documents may come from teaching and research institutions in France or abroad, or from public or private research centers.
L'archive ouverte pluridisciplinaire HAL, est destinée au dépôt et à la diffusion de documents scientifiques de niveau recherche, publiés ou non, émanant des établissements d'enseignement et de recherche français ou étrangers, des laboratoires publics ou privés. 


\title{
Reduction of quantum noise in optical parametric amplification
}

\author{
J. A. Levenson, I. Abram, and Th. Rivera \\ France Telecom, Centre National d'Etudes des Télécommunications, Paris B, BP 107, 92225 Bagneux Cedex, France \\ Ph. Grangier \\ Institut d'Optique, BP 147, 91403 Orsay, France \\ Received November 23, 1992; revised manuscript received April 13, 1993

\begin{abstract}
We experimentally demonstrate that a type-II pulsed optical parametric amplifier operated in a phaseinsensitive configuration works as a near-perfect classical optical amplifier whose noise figure approaches $3 \mathrm{~dB}$ at high gains. We further demonstrate that, when operated in a phase-sensitive configuration, this amplifier works as a quantum-optical amplifier whose noise figure goes below $3 \mathrm{~dB}$ and approaches $0 \mathrm{~dB}$ at high gains. The noise figure of $1.45 \pm 0.2 \mathrm{~dB}$, measured for a gain of $9 \mathrm{~dB}$, is clearly in the quantum regime.
\end{abstract}

\section{INTRODUCTION}

Optical amplifiers (OA's) are a key element in communications systems because they can directly amplify optical signals and are free of the bandwidth limitations imposed on optical communications by conventional electronic amplifiers. Since in optical communications OA's are cascaded in series (as in-line repeaters, as boosters before branching, or as preamplifiers before detection), it is imperative that they do not add noise to the signals that they amplify. We quantify this feature by means of the noise figure (NF) of the amplifier, which is defined as the signal-tonoise ratio (SNR) at the input of the amplifier divided by the SNR at the output, that is,

$$
\mathrm{NF}=\frac{\mathrm{SNR}_{\text {in }}}{\mathrm{SNR}_{\text {out }}}
$$

Clearly, for an ideal amplifier that adds no noise, we must have $\mathrm{NF}=1$ (i.e., $0 \mathrm{~dB}$ ).

In the best traveling-wave amplifiers that are used to date in optical systems, the NF attains a value of $\sim 5 \mathrm{~dB}$ for semiconductor $\mathrm{OA}^{\prime} \mathrm{s}^{1}$ and $\sim 4 \mathrm{~dB}$ for Er-doped opticalfiber OA's. ${ }^{2}$ The NF's of these OA's cannot be improved much further because they approach the quantum limit for phase-insensitive amplification, which is $3 \mathrm{~dB}^{3-6}$ Such a phase-insensitive amplifier (PIA), in addition to amplifying the quadrature that carries the signal, also amplifies the conjugate quadrature that carries only noise. In this case it can be shown ${ }^{3-6}$ (see also Section 2 below) that the SNR is degraded by a factor of 2 , hence the NF of $3 \mathrm{~dB}$. This situation is depicted in Fig. 1(a), in which the beams at the input and output of the PIA are represented in a phase-space diagram. The quantum noise of the coherent input is depicted by a disk of unit radius surrounding the vector extremity. The edge of the disk represents the $1 / e$ isoprobability curve for the possible outcomes of a measurement. The area of the disk is directly given by the Heisenberg uncertainty principle, which for a coherent beam verifies

$$
\Delta E_{1} \Delta E_{2}=1,
$$

where $\Delta E_{1}$ and $\Delta E_{2}$ are in units of $\sqrt{\hbar \omega / 2}$. On phaseinsensitive amplification all the dimensions of the disk are magnified by the same factor. Thus the noise spot remains a disk (i.e., the noise power is the same in both quadratures), but its area is now larger, indicating that the amplifier has introduced excess noise beyond that required by the Heisenberg uncertainty principle.

Thirty years ago Louisell ${ }^{3}$ pointed out that a coupledmode parametric amplifier can overcome this limit if both its input channels are excited. More recently several workers ${ }^{4-8}$ contributed to the development of a theoretical framework for the analysis of quantum noise in optical amplifiers. The main idea is that a parametric amplifier that has both input channels excited is sensitive to the phase of the input signal. Such a phase-sensitive amplifier (PSA) amplifies both the signal and the noise in the quadrature that carries information but deamplifies the noise in the conjugate quadrature that carries no information. In this case it can be shown ${ }^{3}$ (see also Section 2 below) that the SNR remains unchanged, implying that an ideal PSA has $\mathrm{NF}=1$ (i.e., $0 \mathrm{~dB}$ ) in the amplified quadrature. Figure 1(b) allows us to understand this situation better. By amplifying one quadrature and deamplifying its conjugate, one distorts the noise spot into an ellipse, in contrast to the PIA configuration. However, in spite of the distortion, the area of the noise spot is unchanged and remains equal to that required by the Heisenberg uncertainty principle. The amplifier thus operates in the quantum-limited regime. This means that no additional noise is introduced into the signal in the amplified quadrature, thus resulting in a NF of $0 \mathrm{~dB}$, which is associated with quantum-limited optical amplification. Although many quantum features of parametric amplification such as twin-photon-noise reduction, ${ }^{9}$ squeezing, ${ }^{10,11}$ and quantum-nondemolition measurements ${ }^{12}$ have been recently introduced, the early theoretical predictions on the quantum NF of parametric amplification, to our knowledge, have not yet been experimentally demonstrated. 

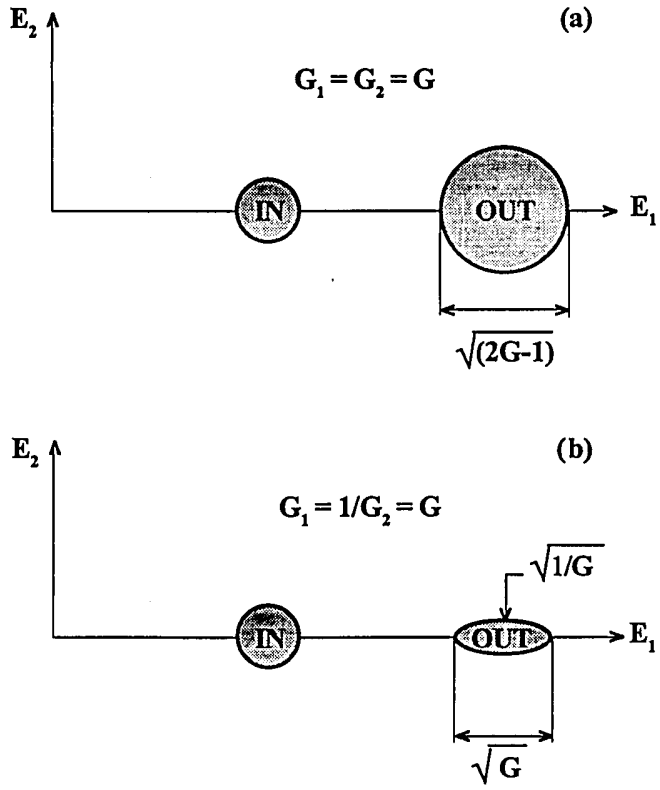

Fig. 1. Phase-space representations of (a) the phase-insensitive and (b) phase-sensitive amplification of a coherent state.

In this paper we experimentally demonstrate that a type-II optical parametric amplifier can act as both a nearperfect classical OA with a minimal $\mathrm{NF}$ of $\sim 3 \mathrm{~dB}$ and a noiseless quantum $\mathrm{OA}$ with a $\mathrm{NF}$ approaching $0 \mathrm{~dB}$ at high gains in the amplified quadrature. To switch between the two types of operation, it suffices to rotate the polarization of the incoming signal and thus change from phaseinsensitive to phase-sensitive amplification, as explained in Section 2 below. The paper is organized as follows. In Section 2 we give an outline of the theory of quantum noise in parametric amplification in both the PIA and the PSA configurations. In Section 3 we present the experiments that we performed to demonstrate the theoretical relationships that are derived in Section 2, and in Section 4 we discuss the experimental results.

\section{THEORY OF THE NOISE FIGURE OF A PARAMETRIC AMPLIFIER}

A type-II parametric amplifier involves two channels, signal and idler (denoted as $a$ and $b$, respectively), which are orthogonally polarized to each other. These two channels are coupled to each other by the pumping process of the amplifier, which produces twin photons, one photon in each channel. One formalizes this coupling by means of the Hamiltonian

$$
H=\frac{\gamma}{v}\left(a^{+} b^{+}+a b\right),
$$

where $a^{+}(a)$ is a boson-creation (-annihilation) operator that acts on the electromagnetic excitation of the signal channel, whereas $b^{+}(b)$ acts on the idler channel. $v$ is the group velocity of light in the nonlinear crystal, and $\gamma=\chi^{(2)} E_{p} \exp \left(i \phi_{p}\right)$ is the pumping parameter of the amplifier, which depends on the medium nonlinearity $\chi^{(2)}$ and on the pump-field amplitude $E_{p}=\sqrt{I_{p}}$. One may regard the pump phase as a reference, so that $\phi_{p}=0$.

When light propagates in the nonlinear crystal it evolves under the Hamiltonian of Eq. (3), so that at the output of the amplifier the operators for the signal channel are related to the input signal and idler operators by ${ }^{5}$

$$
\begin{aligned}
a_{\text {out }} & =\cosh (\gamma z) a+\sinh (\gamma z) b^{+}, \\
a_{\text {out }}{ }^{+} & =\cosh (\gamma z) a^{+}+\sinh (\gamma z) b .
\end{aligned}
$$

The number operator that corresponds to an intensity measurement of the output of the signal channel is

$$
\begin{aligned}
N= & a_{\text {out }}^{+} a_{\text {out }} \\
= & \cosh ^{2}(\gamma z) a^{+} a+\sinh ^{2}(\gamma z) b b^{+} \\
& +\cosh (\gamma z) \sinh (\gamma z)\left(a^{+} b^{+}+a b\right) .
\end{aligned}
$$

We first examine the configuration in which the input beam is polarized parallel to the signal channel, so that only this channel is excited. In this case one can calculate the number of photons measured at the signal output from Eq. (5) as

$$
\langle N\rangle_{\mathrm{PIA}}=\left\langle a_{\text {out }}{ }^{+} a_{\text {out }}\right\rangle=n \cosh ^{2}(\gamma z)+\sinh ^{2}(\gamma z),
$$

where $n$ is the number of photons at the input. In general, $n \gg 1$ even for signals considered to be weak, so that the $\sinh ^{2}(\gamma z)$ term, which represents the spontaneous parametric emission, is neglected compared with the first term. We use this approximation $(n \gg 1)$, whereby one neglects the contribution of the spontaneous parametric emission to the measured intensity, throughout this paper. Thus, according to Eq. (6), the output of the signal channel is amplified with respect to the input with a gain of

$$
G_{\mathrm{PIA}}=\cosh ^{2}(\gamma z),
$$

which is independent of the phase of the input signal. Note that parametric emission also occurs along the idler channel. However, because the polarization of the idler is orthogonal to that of the signal channel, we do not include the idler power in the definition of the gain. The above analysis, in which only the signal channel is being detected, applies to our experimental configuration in which the two channels are separated from each other by use of a polarizer at the output of the amplifier, and only one output channel is detected.

On the other hand, if the input beam is polarized at $45^{\circ}$ with respect to the signal and the idler polarizations, both input channels of the amplifier are excited. The number of photons measured at the output of the signal channel is

$$
\langle N\rangle=\frac{n}{2}[\cosh (2 \gamma z)+\sinh (2 \gamma z) \cos (\phi)],
$$

where $n$ is the total number of photons in the input beam, so that $n / 2$ excite each of two channels. Equation (8) shows that when both input channels are simultaneously excited, the total (signal plus idler) phase-sensitive gain of parametric amplification is

$$
G_{\phi}=\cosh (2 \gamma z)+\sinh (2 \gamma z) \cos (\phi),
$$

where $\phi=\phi_{\text {pump }}-\phi_{\text {signal }}-\phi_{\text {idler }}$ is the sum of the signal and idler phases at the input relative to the pump phase. For the particular phase of the signal and idler for which $\phi_{\text {signal }}=\phi_{\text {idler }}=\phi=0$, the total phase-sensitive parametric gain is maximum and is related to the pumping pa- 
rameter by

$$
G_{0}=\exp (2 \gamma z)
$$

For the other quadrature, $\phi_{\text {signal }}=\phi_{\text {idler }}=\pi / 2$, so that we have $\phi=\pi$, and the gain becomes $<1$ :

$$
G_{\pi}=\exp (-2 \gamma z)=1 / G_{0},
$$

implying that the input is deamplified.

The quantum-noise power that is associated with the two types of amplification discussed above (PIA and PSA) is proportional to the variance of the number of photons in the output beam,

$$
\left\langle\Delta N^{2}\right\rangle=\left\langle N^{2}\right\rangle-\langle N\rangle^{2} .
$$

For the case of PIA in which there is an input to the signal channel alone, one can calculate the noise power from Eq. (12) as

$$
\left\langle\Delta N^{2}\right\rangle_{\text {PIA }}=n \cosh ^{2}(\gamma z)\left[\cosh ^{2}(\gamma z)+\sinh ^{2}(\gamma z)\right],
$$

which for the output SNR gives

$$
(\mathrm{SNR})_{\mathrm{out}}=\frac{\alpha n \cosh ^{2}(\gamma z)}{\cosh ^{2}(\gamma z)+\sinh ^{2}(\gamma z)},
$$

where we use the definition of SNR in terms of noise power and introduce the proportionality constant $\alpha$ that converts the photon number into power, and thus its value depends on the units used in the measurement. Since the SNR at the input of the amplifier for a shot-noise-limited beam is proportional to the number of photons in the beam:

$$
(\mathrm{SNR})_{\mathrm{in}}=\alpha n,
$$

the NF of the PIA is

$$
\mathrm{NF}_{\mathrm{PIA}}=\frac{\cosh ^{2}(\gamma z)+\sinh ^{2}(\gamma z)}{\cosh ^{2}(\gamma z)}=2-\frac{1}{G_{\mathrm{PIA}}},
$$

which for large gains approaches $\mathrm{NF}_{\mathrm{PIA}}=2$ (or $3 \mathrm{~dB}$ ).

On the other hand, for the case in which both the signal and the idler inputs are excited and thus the parametric amplification becomes phase sensitive, one calculates the noise power from Eq. (14) as

$$
\langle\Delta N\rangle_{\phi}=\frac{n}{2}[\cosh (2 \gamma z)+\sinh (2 \gamma z) \cos (\phi)] \cosh (2 \gamma z),
$$

which, for the NF of the phase-sensitive amplifier, gives

$$
\mathrm{NF}_{\phi}=\frac{2 \cosh (2 \gamma z)}{(\cosh (2 \gamma z)+\sinh (2 \gamma z) \cos (\phi)}=\frac{G_{0}+\left(1 / G_{0}\right)}{G_{\phi}}
$$

In the amplified quadrature $(\phi=0), \mathrm{NF}_{\phi}$ takes the limit

$$
\mathrm{NF}_{0}=1+\frac{1}{{G_{0}}^{2}}
$$

Clearly, at large gains the NF in the amplifying quadrature approaches $\mathrm{NF}_{0}=1$, indicating noiseless amplification. In the deamplified quadrature, on the other hand $(\phi=\pi), \mathrm{NF}_{\pi}=1+G_{0}{ }^{2}$, which implies that excess noise is added at all the gains.
In the above analysis we chose the case in which only one of the outputs of the amplifier is detected (e.g., the signal channel), which corresponds to an experimental configuration similar to that of the PIA in which the signal and idler channels are separated from each other. Thus, when the amplifier is not pumped so that its gain is unity $\left(G_{0}=1\right)$, Eq. (19) gives a NF that is equal to 2 . This occurs because when only the signal channel is detected half the input energy is lost, and this degrades the SNR by a factor of 2 for a shot-noise-limited beam. Equation (19) shows that, at very large gain, the NF becomes 1 and the input SNR is recovered after amplification. This means that the PSA does not introduce any additional noise, whereas at the same time it compensates the partition noise produced by the $50 \%$ loss at the output..$^{13}$

Note that the beams polarized at $45^{\circ}$ with respect to the crystal axes are the eigenmodes of two independent degenerate parametric amplifiers even though the signal and idler channels are always nondegenerate because of their orthogonal polarizations. One can readily see this by considering the operators

$$
d_{ \pm}=(a \pm b) / \sqrt{2}
$$

which correspond to the two $45^{\circ}$-oriented polarizations. These operators transform the nondegenerate parametric Hamiltonian [Eq. (3)] into the Hamiltonian for two independent degenerate parametric amplifiers:

$$
H=\frac{\gamma}{2 v}\left(d_{+}{ }^{+} d_{+}{ }^{+}+d_{+} d_{+}\right)-\frac{\gamma}{2 v}\left(d_{-}{ }^{+} d_{-}^{+}+d_{-} d_{-}\right),
$$

implying that in the course of amplification the modes that are polarized at $45^{\circ}$ will undergo squeezing and will have their noise reduced. If the full output of the parametric amplifier is detected (signal plus idler or $d_{+}+d_{-}$), then the total number of photons is

$$
\langle N\rangle_{\text {TOTAL }}=n[\cosh (2 \gamma z)+\sinh (2 \gamma z) \cos (\phi)],
$$

whereas the corresponding noise is

$$
\left\langle\Delta N^{2}\right\rangle_{\mathrm{TOTAL}}=n[\cosh (4 \gamma z)+\sinh (4 \gamma z) \cos (\phi)],
$$

which indicates that the NF for the total output (signal plus idler) of the phase-sensitive parametric amplifier in the amplified quadrature $(\phi=0)$ is always

$$
\mathrm{NF}_{0}=1 \text {, }
$$

independently of the PSA gain.

We now return to our analysis of the situation that corresponds to our experimental configuration, in which only one of the output channels is detected. One can take into account the effects of downstream losses (other than the $50 \%$ loss that is associated with the detection of a single channel, as discussed above) by using the familiar inputoutput relationship for an attenuator ${ }^{5}$ with a loss coefficient $(1-\eta)$

$$
a_{\mathrm{out}}=\sqrt{\eta} a_{\mathrm{in}}+\sqrt{1-\eta} c_{\mathrm{in}}
$$

where $c_{\text {in }}$ is an unexcited channel that introduces only quantum noise. Combining the input-output relationships for the parametric amplifier and for the attenuator and using Eqs. (5) and (25), one can write the number operator for the photons that reach the detector as 


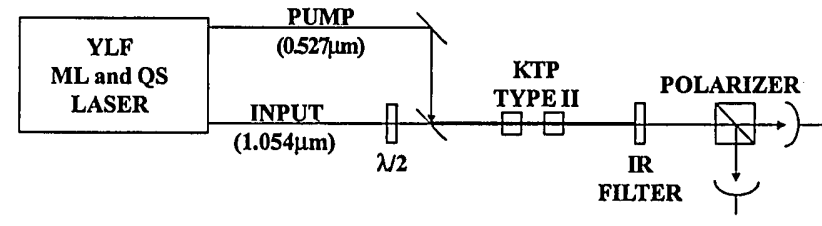

NOISE-MEASUREMENT CHANNEL (18MHz)

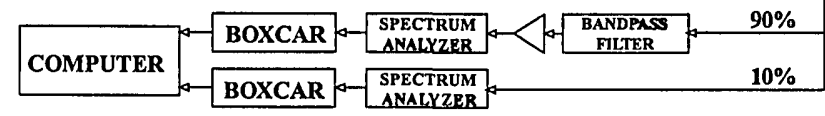

SIGNAL - MEASUREMENT CHANNEL (76MHz)

Fig. 2. Experimental setup. A frequency-doubled mode-locked (ML) Q-switched (QS) YLF:Nd laser pumps (at $527 \mathrm{~nm}$ ) a traveling-wave parametric amplifier composed of two type-II KTP crystals. The polarization of the input signal (at $1054 \mathrm{~nm}$ ) is rotated by the half-wavelength plate $(\lambda / 2)$ to achieve phaseinsensitive or phase-sensitive amplification. The photodiode current is split $90 / 10$, with each partial current measured by use of a spectrum analyzer and a boxcar combination.

$$
\begin{aligned}
N_{\text {det }}= & \eta\left[\cosh ^{2}(\gamma z) a^{+} a+\sinh ^{2}(\gamma z) b b^{+}\right. \\
& \left.+\cosh (\gamma z) \sinh (\gamma z)\left(a^{+} b^{+}+a b\right)\right]+(1-\eta) c^{+} c \\
& +\sqrt{\eta(1-\eta)}\left[\cosh (\gamma z) a^{+} c+\sinh (\gamma z) b c\right. \\
& \left.+\cosh (\gamma z) a c^{+}+\sinh (\gamma z) b^{+} c^{+}\right]
\end{aligned}
$$

For the case of a PIA the detected signal is therefore

$$
\langle N\rangle_{\text {det }}=\eta n \cosh ^{2}(\gamma z)=\eta n G_{\mathrm{PIA}},
$$

whereas the noise power is

$$
\begin{aligned}
\left\langle\Delta N^{2}\right\rangle_{\mathrm{det}} & =\eta n \cosh ^{2}(\gamma z)\left[1+2 \eta \sinh ^{2}(\gamma z)\right] \\
& =2 \eta^{2} n G_{\mathrm{PIA}}+(1-2 \eta) G_{\mathrm{PIA}}{ }^{2} .
\end{aligned}
$$

This corresponds to an overall NF of

$$
\mathrm{NF}_{\mathrm{PIA}}=2-\frac{2}{G_{\mathrm{PIA}}}+\frac{1}{\eta G_{\mathrm{PIA}}},
$$

which shows that at low gains the presence of downstream losses degrades the NF of the amplifier but that at high gains this degradation is compensated and that one attains the classical 3-dB limit.

Similarly, for the case of PSA, the output of the signal channel is

$$
\langle N\rangle_{\phi}=\eta \frac{n}{2} G_{\phi},
$$

whereas the noise power in this channel is

$$
\langle\Delta N\rangle_{\phi}=\eta \frac{n}{2} G_{\phi}[\eta \cosh (2 \gamma z)+1-\eta],
$$

corresponding to a NF of

$$
\mathrm{NF}_{\phi}=\frac{2[\eta \cosh (2 \gamma z)+1-\eta]}{\eta G_{\phi}}
$$

In the amplifying quadrature $(\phi=0)$ the NF becomes

$$
\mathrm{NF}_{0}=1+\frac{1}{G_{0}^{2}}+\frac{2(1-\eta)}{n G_{0}}
$$

Clearly, at high gain the noise introduced by downstream losses is compensated, and the PSA may reach the ideal
NF of 1, a factor of 2 better than the PIA. The experiments presented in this paper aim to demonstrate Eqs. (28) and (33).

\section{EXPERIMENT}

Our experimental setup, schematically presented in Fig. 2, is based on a frequency-doubled mode-locked $Q$-switched YLF laser pumping two type-II KTP crystals in series, constituting a traveling-wave parametric amplifier. The pump beam, at $527 \mathrm{~nm}$, consists of a train of 35-ps-long pulses with a repetition rate of $76 \mathrm{MHz}$ modulated into Gaussian envelopes of 400-ns duration (FWHM), produced at a repetition rate of $400 \mathrm{~Hz}$. A small part of the fundamental (nondoubled) laser beam at $1054 \mathrm{~nm}$ is collinearly injected into the pump beam and serves as the input signal to the amplifier. This beam consists of 630-ns-long (FWHM) Gaussian trains of 50-ps-long pulses, synchronized with the pulses of the pump beam. At the exit of the amplifier one eliminates the pump beam by means of two RG 715 filters and places a polarizer to select the two polarizations (signal and idler) parallel to the crystal axes. Note that the signal and idler outputs of the type-II parametric amplifier are degenerate with respect to wavelength but are clearly nondegenerate (and therefore separable) with respect to polarization. One places a half-wave plate before the amplifier, which permits rotation of the polarization of the input beam. When the input beam is polarized parallel to one of the crystal axes (say, the signal axis) the parametric amplifier is not sensitive to the phase of the input, as shown in Section 2 above: the phase of the idler automatically adjusts itself to any fluctuations of the phase of the signal. On the other hand, when the input is polarized at $45^{\circ}$ with respect to the crystal axes the amplifier is sensitive to the phase of the input signal. For a particular phase $\phi=0$ the light produced by the parametric amplifier is in phase with the input signal and thus constructively interferes with it, giving rise to amplification, whereas for the conjugate quadrature the field emitted by the amplifier is out of phase with the input signal, and thus their interference produces deamplification of the input.

In our experimental setup one adjusts the confocal parameter of all the beams to be much larger than the crystal length to minimize mode distortion and diffraction effects. ${ }^{14}$ One keeps the pump beam to a maximum intensity of $50 \mathrm{MW} / \mathrm{cm}^{2}$, also to minimize distortion, thus producing a maximum power gain of $G_{\mathrm{PIA}}$ of $6.4 \mathrm{~dB}$ in the phase-insensitive configuration and $G_{0}$ of $9 \mathrm{~dB}$ in the amplified quadrature of the phase-sensitive configuration.

The output of the signal channel is focused (FWHM = $80 \mu \mathrm{m}$ ) onto a $300-\mu \mathrm{m}$-diameter InGaAs photodiode (Epitaxx ETX-300) with nominal quantum efficiency of $\sim 90 \%$ and low dark current. One avoids optical saturation of the photodiode by limiting the optical gain and by adjusting the input-signal intensity to be of the order of $20 \mathrm{~kW} / \mathrm{cm}^{2}$, so that the peak photocurrent never exceeds $10 \mathrm{~mA}$, i.e., $0.5 \mathrm{~V}$ on $50 \Omega$. The output photocurrent from the photodiode is split $90 / 10$, and the $10 \%$ portion is introduced into a spectrum analyzer (HP 8563A) set at $76 \mathrm{MHz}$ : measurement of the $76-\mathrm{MHz}$ modulation of the mode-locked laser constitutes the signal carried by the beam. The $90 \%$ portion of the photocurrent is introduced 


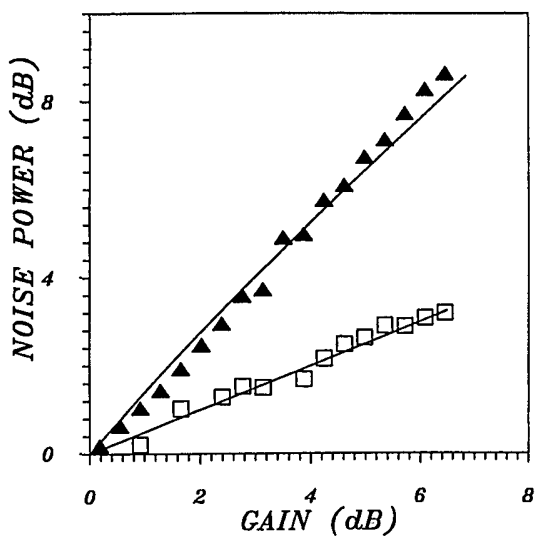

Fig. 3. Noise power of the parametric output in the phaseinsensitive configuration as a function of gain (triangles), fitted to Eq. (28). The squares represent the measured noise power of a coherent beam.

into a bandpass filter that transmits between 12 and $25 \mathrm{MHz}$ and has $90-\mathrm{dB}$ attenuation outside this range. This filter prevents saturation of the subsequent low-noise amplifier (Trontech W110B-13) by the 76-MHz modulation and its harmonics. We checked this by examining the absence of distortion in the temporal profile of the amplified pulse. The amplifier output is fed into a second spectrum analyzer with $10-\mathrm{MHz}$ rf bandwidth (Tektronix 2782) set at $18 \mathrm{MHz}$. In principle, in the determination of the SNR, one must measure the noise over the same frequency band as the signal. However, because of the very large amplitude of the signal at $76 \mathrm{MHz}$, direct measurement of the quantum noise at this frequency is not possible. On the other hand, measurement of the noise at $18 \mathrm{MHz}$ gives a quantitative measure of the quantum noise at the modulation frequency $(76 \mathrm{MHz})$ because of the large bandwidth of traveling-wave pulsed parametric amplification $(>100 \mathrm{GHz})$. The large bandwidth of the spectrum analyzer (and bandpass filter) permits one to time resolve the envelope of the $Q$-switched pulse train, and only approximately seven mode-locked pulses at the center of the train are effectively registered (50-ns time window).

To treat the signals quantitatively, video averaging is provided by two boxcars following each of the two spectrum analyzers; the boxcars are triggered by the $Q$-switch synchronization from the laser-power supply, and their gate width is $50 \mathrm{~ns}$. Each measurement involves $\sim 5000$ $Q$-switched trains, which are averaged by a computer that receives the data from the boxcars. One performs all the measurements by simultaneously registering the modulation (at $76 \mathrm{MHz}$ ) and the noise (at $18 \mathrm{MHz}$ ) for the output beams. These two quantities are then plotted as $x$ and $y$, respectively, giving the noise power as a function of the amplifier gain. In the case of the PIA, one varies the gain $G_{\text {PIA }}$ by changing the incident intensity of the pump beam. For the case of PSA, one varies the gain $G_{\phi}$ by scanning the relative phase of the pump and input beams. One achieves this by modulating the path of the input beam with a piezoelectric transducer.

\section{RESULTS}

First we consider the PIA configuration in which the polarization of the input beam is fixed parallel to the ex- traordinary axis of the crystal; this polarization corresponds to the signal channel.

The results of the measurement of quantum-noise power in the PIA configuration are given in Fig. 3, in which the measured noise (triangles) is plotted as a function of the amplifier gain $G_{\text {PIA }}$, expressed in decibels. One obtains the 0 -dB point by removing the pump beam, and therefore this point corresponds to $G_{\mathrm{PIA}}=1$ and is then followed by an attenuation $(1-\eta)$ resulting from the overall detection efficiency $\eta$. The theoretical expression [Eq. (28) divided by $\eta$ is also plotted for $\eta=0.85$, which fits the experimental data quite well. In Fig. 3 are also plotted, for comparison, the noise power resulting from shot noise at each output intensity (squares). One obtains these data by turning off the pump beam and then adjusting the intensity of the input laser beam so that it matches the output intensity measured in the presence of amplification. As expected, the shot-noise curve is a line of slope 0.5. Note that at all the output intensities the noise power of the amplified beam is larger than the noise power corresponding to shot noise. In Fig. 4 we plotted the NF that can be deduced from the experimental data (triangles) and fitted it to Eq. (29). Note that for a gain of $G_{\text {PIA }}$ of $6.4 \mathrm{~dB}$, the largest examined in this experiment, we obtained a $\mathrm{NF}$ of $2.2 \mathrm{~dB}$, whereas at larger gains the NF should approach $3 \mathrm{~dB}$, as the theoretical curve of Eq. (29) indicates.

We now consider the experimental PSA configuration, in which the polarization of the input beam is set at $45^{\circ}$ with respect to the extraordinary and ordinary axes, so that it projects equally onto the signal and idler polarizations. As discussed in Section 2 above, the parametric amplifier is phase sensitive in this configuration. Note that, in this experiment, to minimize the changes between the PIA and the PSA configurations, one retains the polarizer separating signal and idler at the output of the amplifier.

The noise power measured in the phase-sensitive configuration is presented in Fig. 5, where it is plotted as a function of the phase-sensitive gain $G_{\phi}$ in decibels. The experimental results (stars) are quite well fitted by the expected theoretical expression [Eq. (31)], with $\eta=0.82$. The shot noise that corresponds to the output intensity is also plotted (squares). In this configuration it was possible to check whether the input beam was shot-noise lim-

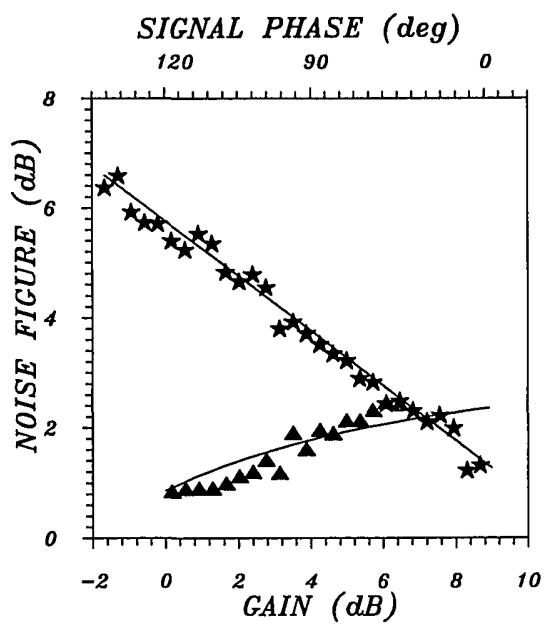

Fig. 4. NF of the phase-insensitive (triangles) and phasesensitive (stars) parametric amplifiers, fitted to Eqs. (29) and (33), respectively. 


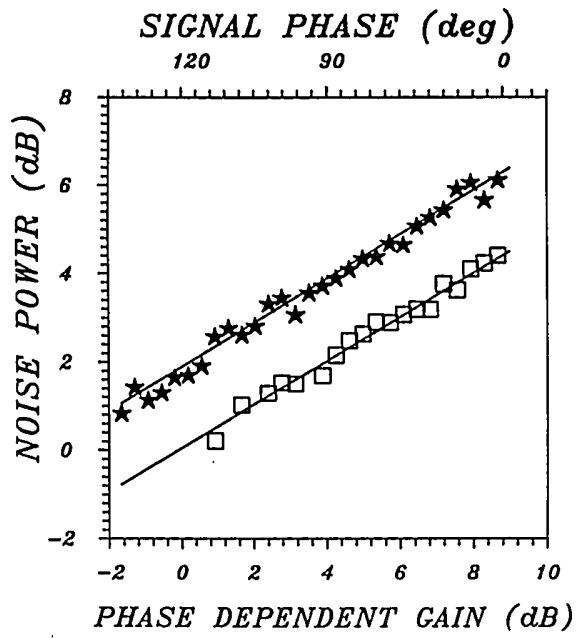

Fig. 5. Noise power of the parametric output in the phasesensitive configuration as a function of phase-sensitive gain (stars), fitted to Eq. (31). The squares represent the measured noise power of a coherent beam.

ited by detecting both output channels (signal and idler) and by verifying that, in the absence of the pump beam, the noise of the sum and difference of the two photocurrents gave identical results. As in the PIA configuration, the amplified beam is noisier than is a coherent beam of equivalent intensity. As one can see by comparing Eqs. (30) and (31), the relation between the shot noise and the noise of the parametric-amplifier output is a multiplicative factor $>1$, independent of phase, and thus the plot of Eq. (31) is a line of slope 0.5 lying above the shotnoise line: at each output intensity the amplified beam is noisier than is a shot-noise-limited beam of the same intensity, partly because the input noise is being amplified and partly because the amplifier adds noise. To distinguish between these two effects, we plotted in Fig. 4 above the NF of the PSA, as a function of the gain $G_{\phi}$ in decibels. Note that at low gains examined in this experiment $\left(G_{\phi}\right.$ up to $\left.9 \mathrm{~dB}\right)$ the amplifier adds some noise. However, at the phases that correspond to $G_{\phi}>7 \mathrm{~dB}$, the noise added is significantly less than that of a classical amplifier, as can be seen in Fig. 4 above, indicating that the amplifier operates in the quantum regime. More particu- larly, in the amplified quadrature $(\phi=0)$ that displays a gain $G_{\phi}$ of $9 \mathrm{~dB}$, the amplifier achieves an $\mathrm{NF}_{0}$ of $1.45 \pm$ $0.2 \mathrm{~dB}$, clearly lower than the NF of a PIA of equivalent gain (and losses), which is $2.4 \pm 0.2 \mathrm{~dB}$.

\section{CONCLUSION}

We have presented experimental results on the noise figure of a type-II pulsed parametric amplifier. We have shown that, in the relatively low gains examined in these experiments $(<9 \mathrm{~dB})$, in both phase-insensitive and phasesensitive amplification this device achieves the theoretical performances of a near-perfect classical and quantum amplifier, respectively. These results indicate that at higher gains a PIA parametric amplifier achieves the ideal noise figure of $3 \mathrm{~dB}$, whereas in the phase-sensitive configuration it attains a noise factor of $0 \mathrm{~dB}$, underscoring the potential interest that such parametric amplifiers may present in long-haul optical-communications systems. The phase-sensitive configuration with its near-unity noise figure is clearly more advantageous, but its practical implementation is much more difficult because of the problems that are associated with control of the signal phase.

\section{REFERENCES}

1. T. Saitoh and T. Mukai, IEEE J. Quantum Electron. QE-23, 1010 (1987).

2. M. J. Petit, R. A. Backer, and A. Hadjifotiou, Electron. Lett. 25, 273 (1989).

3. W. H. Louisell, Coupled Mode and Parametric Electronics (Wiley, New York, 1960); Radiation and Noise in Quantum Electronics (McGraw-Hill, New York, 1964).

4. C. M. Caves, Phys. Rev. D 26, 1817 (1982).

5. H. P. Yuen, Phys. Rev. Lett. 56, 2176 (1986).

6. Y. Yamamoto, S. Machida, S. Saito, N. Imoto, T. Yanagawa, M. Kitagawa, and G. Bjork, Prog. Opt. 28, 87 (1990).

7. M. J. Collett and D. F. Walls, Phys. Rev. Lett. 61, 2442 (1988).

8. N. C. Wong, Opt. Lett. 16, 1698 (1991).

9. O. Aytur and P. Kumar, Phys. Rev. Lett. 65, 1551 (1990).

10. R. E. Slusher, P. Grangier, A. LaPorta, B. Yurke, and M. J. Potasek, Phys. Rev. Lett. 59, 2566 (1987).

11. P. D. Townsend and R. Loudon, Phys. Rev. A 45, 458 (1992).

12. A. Laporta, R. E. Slusher, and B. Yurke, Phys. Rev. Lett. 62, 28 (1989).

13. J. A. Levenson, I. Abram, Th. Rivera, Ph. Fayolle, J. C. Garreau, and Ph. Grangier, Phys. Rev. Lett. 70, 267 (1993).

14. A. LaPorta and R. E. Slusher, Phys. Rev. A 44, 2013 (1991). 